\title{
DIAGNÓSTICO DA QUALIDADE AMBIENTAL DOS LAGOS E NASCENTES DO ZOOLÓGICO DE GUARULHOS, MUNICÍPIO DE GUARULHOS -SP
}

\section{DIAGNOSIS OF ENVIRONMENTAL QUALITY OF LAKES AND SPRINGS OF THE GUARULHOS ZOO, GUARULHOS-SP}

\author{
Luís Henrique Nunes de Souza1', Regina de Oliveira Moraes Arruda², Edna Ferreira Rosini ${ }^{3}$, \\ Darah Danielle Pontes ${ }^{4}$
}

\section{RESUMO}

O crescimento urbano desordenado tem causado degradação ambiental e as áreas de proteção ambiental estão diminuindo. Assim, o uso e ocupação da terra têm trazido inúmeros problemas socioambientais. Com esse princípio em mente, locais como o Zoológico de Guarulhos, umas das principais áreas verdes de laser do município devem ser preservadas, principalmente por conta da existência de uma nascente dentro dos limites do zoológico e pelo fato de suas águas serem usadas para alimentar todos os lagos dentro dos viveiros dos animais e outros fins. O objetivo deste trabalho foi avaliar a qualidade das águas dos lagos e nascente do Zoológico de Guarulhos-SP, através do índice do estado trófico (IET). Para alcançar os objetivos propostos foi realizada uma avaliação do estado de preservação da nascente e coletas de água em quatro pontos, iniciando pela nascente e dois lagos dentro do Zoológico e um ponto já na parte urbanizada do córrego Ana Rita. As coletas obedeceram aos parâmetros de preservação e transporte, estabelecidos pela Agência Nacional de Águas. No laboratório foram realizadas as análises de turbidez, Demanda Bioquímica de Oxigênio (DBO), Clorofila a, Fósforo Total (PT) Nitrogênio Total (NT) e Escherichia coli (E. coli) e in loco avaliou-se temperatura, $\mathrm{pH}$, condutividade, Oxigênio Dissolvido (OD). A partir das análises foi possível averiguar que do primeiro ponto de coleta ao último, à montante daquele, há um aumento da eutrofização das águas, iniciando como classe supereutrófico e ao final chegou a hipereutrófico. Foi realizada também a avaliação ambiental da nascente e ela foi considerada como ruim a péssima devido às condições desfavoráveis para sua preservação, necessitando de uma atenção especial. Os resultados obtidos devem-se ao acúmulo de nutrientes ao longo do córrego, tanto de origem biológica, a partir da matéria produzida nos viveiros que compõem o zoológico, quanto de origem antrópica, como esgoto não tratado, e descarte incorreto de material no rio, em seus pontos não protegidos, esse fato foi atestado pela grande presença de E. coli. Quanto à avaliação ambiental da nascente, ela foi considerada como ruim a péssima devido às condições desfavoráveis para sua preservação.

PALAVRAS-CHAVE: Análise Geoambiental. Qualidade de água. Zoológico Municipal de Guarulhos. Microbiológica. Índice do Estado Trófico (IET).

\section{ABSTRACT}

The disordered urban growth has caused environmental degradation and the environmental protected areas are decreasing. Thus the use and occupation of the land has brought numerous social and environmental problems. With this principle in mind, places such as Guarulhos Zoo, one of the municipality's main green laser areas, should be preserved mainly because of the existence of a spring within the zoo's boundaries and that its waters are used to feed all lakes within animal nurseries and other purposes. The objective of this work was to evaluate the quality of the lakes and spring waters of the Guarulhos Zoo - SP, through the trophic state index (EIT). To achieve the proposed objectives, an assessment of the state of preservation of the spring and

1 Discente do curso de Farmácia, participante do Programa de Iniciação Científica - PIBIC-UNG da Universidade UNG.

2 Docente da graduação e do Mestrado em Análise Geoambiental da Universidade UNG.

3 Docente da graduação e do Mestrado em Análise Geoambiental da Universidade UNG.

4 Discente do curso de Ciências Biológicas, participante do Programa de Iniciação Científica PIBIC-UNG da Universidade UNG. 
water collections was performed at four points, starting with the spring and two lakes within the Zoo and one point already in the urbanized part of Ana Rita stream. The collections obeyed the preservation and transportation parameters, established by the National Water Agency. Turbidity, Biochemical Oxygen Demand (BOD), Chlorophyll a, Total Phosphorus (PT) Total Nitrogen (NT) and Escherichia coli (E. coli) analyzes were performed in the laboratory and temperature, $\mathrm{pH}$, conductivity, Dissolved Oxygen (DO). From the analysis it was possible to verify that from the first collection point to the last there is an increase of the water eutrophication, starting as a supereutrophic class and at the end it reached hypereutrophic. The environmental assessment of the spring was also performed and it was considered as bad to very bad due to unfavorable conditions for its preservation, requiring special attention. The results obtained are due to the accumulation of nutrients along the stream, both of biological origin, from the material produced in the nurseries that make up the zoo, and we also observed materials of anthropic origin such as untreated sewage and incorrect disposal of material in the zoo. In its unprotected points, this fact was attested by the large presence of E. coli. As for the environmental assessment of the spring, it was considered as bad to very bad due to unfavorable conditions for its preservation, needing special attention.

KEYWORDS: Geoenvironmental Analysis. Water Quality. Guarulhos Municipal Zoo. Microbiological. Trophic State Index (EIT). 


\section{INTRODUÇÃO}

Com a degradação ambiental, as áreas de proteção estão diminuindo, e o uso e ocupação da terra trazido inúmeros problemas socioambientais. Como as ocupações irregulares que despejam resíduos sólidos e líquidos de maneira irregular, atingindo assim as bacias hidrográficas, na maioria dos casos degradando a nascente (TUCCI, 2010).

Conforme a resolução CONAMA 001/86 (BRASIL, 1986): "Impacto ambiental é qualquer alteração das propriedades físicas, químicas e biológicas do meio ambiente, causada por qualquer forma de matéria ou energia resultante das atividades humanas que, direta ou indireta, afetam: a saúde, a segurança e o bem-estar da população; as atividades sociais e econômicas; a biota; as condições estéticas e sanitárias do meio ambiente; a qualidade dos recursos ambientais"

De acordo com a legislação $n^{\circ} 12.651 / 2012$ (BRASIL, 2012), a Área de Preservação Permanente - APP corresponde a uma área protegida, coberta ou não por vegetação nativa, cuja função ambiental é a de preservar os recursos hídricos, a paisagem, a estabilidade geológica e a biodiversidade, facilitar o fluxo gênico de fauna e flora, proteger o solo e assegurar o bem-estar das populações humanas. Nascente é o afloramento natural do lençol freático e que dá início a um curso d'água. Perene, por seu turno, quer dizer aquilo que não tem interrupção (ROCCO, 2015).

No Brasil, as principais fontes de degradação dos recursos de água doce são esgotos domésticos e industriais, que são lançados nos corpos de água continentais em quase sua totalidade sem nenhuma forma de tratamento (ESTEVES, 2011).

\section{OBJETIVO}

O objetivo do trabalho foi avaliar a qualidade das águas do córrego Ana Rita, a partir da nascente no Zoológico de Guarulhos-SP e a qualidade ambiental da nascente em relação à eutrofização.

\section{METODOLOGIA}

\subsection{Coleta de Amostras}

Foram selecionados quatro pontos de coleta no córrego Ana Rita. Sendo o primeiro ponto na nascente, o segundo e terceiro pontos nos lagos dentro do Zoológico. O quarto ponto que está localizado na parte externa ao zoológico a aproximadamente 100 metros de seus limites (Figura 1)..
Pelo decreto no 10.755 , de 22 de novembro de 1977 (SÃO PAULO, 1977), o Córrego Ana Rita (2326'43.1"S 46³3'56.9"W Localização obtida pelo Google Earth Pro), que pertence a Sub-bacia do Rio Cabuçu de Cima, foi considerado Classe 1.

Figura 1 - Localização dos pontos de coleta no zoológico Municipal de Guarulhos-SP e entorno.

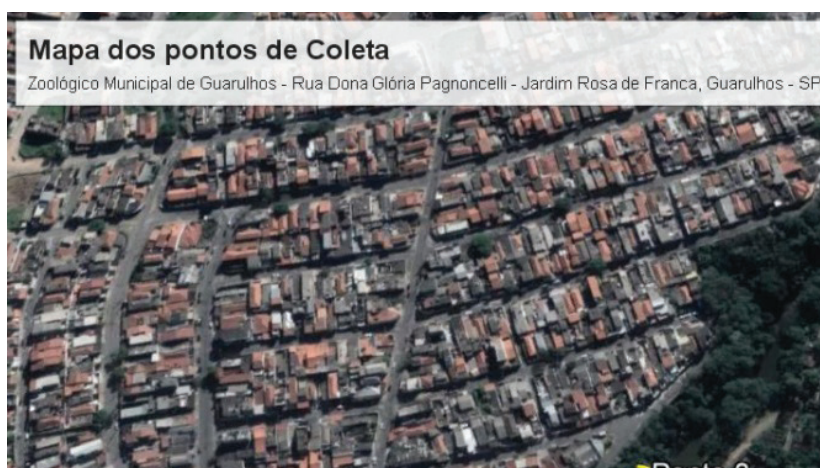

Fonte: imagem obtida através do programa de computador Google Earth Pro

Foram realizadas quatro coletas, uma em cada estação do ano. Em todas as coletas, os procedimentos de armazenagem e preparo, assim como os frascos utilizados e todo manuseio das amostras, seguiram as normas estabelecidas pela Agência Nacional de Águas (ANA, 2019).

\subsubsection{Análises Físico-Químicas}

No campo foram realizadas análises das águas através de instrumentos analíticos devidamente calibrados e com medições em triplicata para os seguintes parâmetros físico-químicos: temperatura $(T)$ (através do aparelho condutivímetro Digimed DM-3), $\mathrm{pH}$ (pHmetro Digimed DM-2), condutividade (CE) (Condutivímetro Digimed DM-3), oxigênio dissolvido (OD) (Oxímetro Digimed DM-4), turbidez (TU) (Turbidímetro Quimis Q279P). No laboratório foram realizadas as análises de fósforo total (PT), nitrogênio total (NT) e Escherichia coli (E. coli) segundo Standard Methods for examinations of Water and Wastewater (APHA, 2012). Para clorofila a metodologia adaptada de WETZEL \& LIKENS (1991) e a demanda bioquímica de oxigênio (DBO), foram utilizados analisadores eletrônicos de DBO via método manométrico (DBO Sensor VELP).

\subsubsection{Avaliação Ambiental da Nascente}

Para a avaliação da qualidade ambiental da nascente urbana, foi utilizado o Guia de Avaliação da 
Qualidade das Águas, adaptado de GOMES; MELO; VALE, (2005), segundo o mesmo, esta análise tem como objetivo avaliar o a influência das áreas urbanas sobre a nascente seguindo parâmetros macroscópicos, tendo como medir o grau de impacto e interferência sobre os mesmos, quadros 1 e 2 .

Quadro1 - Metodologia do índice de impacto ambiental macroscópico para nascente

\begin{tabular}{|c|c|c|c|}
\hline \multirow{2}{*}{$\begin{array}{c}\text { Parâmetro } \\
\text { Macroscópico }\end{array}$} & 3 & 2 & 1 \\
\cline { 2 - 4 } Cor da água & Transparente & Clara & Escura \\
\hline Odor & Inexistente & Fraco & Forte \\
\hline Lixo ao redor & Inexistente & Pouco & Muito \\
\hline $\begin{array}{c}\text { Materiais } \\
\text { flutuantes }\end{array}$ & Inexistente & Pouco & Muito \\
\hline Espumas & Inexistente & Pouco & Muito \\
\hline Óleos & Inexistente & Pouco & Muito \\
\hline Esgoto & Inexistente & Substancial & Presente \\
\hline $\begin{array}{c}\text { Vegetação } \\
\text { presentes }\end{array}$ & Presente & Degradada \\
\hline $\begin{array}{c}\text { Uso por humano Não detectado } \\
\text { Uso por animais }\end{array}$ & Eão detectado & Esporádico & Constante \\
\hline $\begin{array}{c}\text { Acesso } \\
\text { Proximidade } \\
\text { com área } \\
\text { urbana (metros) }\end{array}$ & Mais de 100 & Entre 50 a 100 & Constante \\
\hline
\end{tabular}

Fonte: Adaptado de GOMES; MELO; VALE, (2005)

Quadro 2 - Classificação do grau de preservação da nascente, seguindo os dados de análises macroscópicos.

\begin{tabular}{|l|c|}
\hline Grau de Preservação & Pontuação Final (pontos) \\
\hline Ótima & Entre 37 e 39 \\
\hline Boa & Entre 34 e 36 \\
\hline Razoável & Entre 31 a 33 \\
\hline Ruim & Entre 28 a 30 \\
\hline Péssimo & Abaixo de 28 \\
\hline
\end{tabular}

Fonte: Adaptado de GOMES; MELO; VALE, (2005)

\section{2. Índice do Estado Trófico}

O índice do estado trófico (IET), é o método de classificar os diferentes níveis de qualidade do enriquecimento por nutrientes presentes na água ou o nível trófico assim por se dizer, que está diretamente relacionado ao crescimento de plantas aquáticas e fitoplacton (ANA, 2019), quadro 3.
Quadro 3 - Categorias dos estados trófico da água.

\begin{tabular}{|c|c|c|}
\hline Valor do IET & $\begin{array}{l}\text { Classes } \\
\text { de Estado } \\
\text { Trófico }\end{array}$ & Descrição \\
\hline$=47$ & $\begin{array}{l}\text { Ultraolig- } \\
\text { otrófico }\end{array}$ & $\begin{array}{l}\text { Massa d'água límpida, com } \\
\text { presença muito baixa de nutrientes, } \\
\text { sem estragos para o uso água. }\end{array}$ \\
\hline $47<\mathrm{IET}=52$ & Oligotrófico & $\begin{array}{l}\text { Massa d'água límpida, com baixa } \\
\text { presença de nutrientes, mas não o } \\
\text { suficiente para causar danos para o } \\
\text { uso de tal água. }\end{array}$ \\
\hline $\begin{array}{l}52<\mathrm{IET}= \\
59\end{array}$ & Mesotrófico & $\begin{array}{l}\text { Massa d'água com presença } \\
\text { intermediária de nutrientes, com } \\
\text { potencialmente danos sobre a } \\
\text { qualidade da água, porém em níveis } \\
\text { moderados. }\end{array}$ \\
\hline $59<\mathrm{IET}=63$ & Eutrófico & $\begin{array}{l}\text { Massa d'água com alta presença } \\
\text { de nutrientes comparada ao estado } \\
\text { natural, sua limpidez é afetada por } \\
\text { atividades antrópicas, onde são } \\
\text { causadas alterações indesejáveis } \\
\text { na qualidade da água, interferências } \\
\text { nos seus usos cotidianos. }\end{array}$ \\
\hline $63<\mathrm{IET}=67$ & $\begin{array}{l}\text { Supere- } \\
\text { utrófico }\end{array}$ & $\begin{array}{l}\text { Massa d'água com alta presença } \\
\text { de nutrientes comparada ao } \\
\text { estado natural, de baixa limpidez, } \\
\text { geralmente causadas por ativi- } \\
\text { dade antrópicas, onde alterações } \\
\text { indesejáveis na qualidade da água } \\
\text { ocorrem como o crescimento de } \\
\text { algas, interferindo nos seus usos } \\
\text { cotidianos. }\end{array}$ \\
\hline$>67$ & $\begin{array}{l}\text { Hipereutrófi- } \\
\text { co }\end{array}$ & $\begin{array}{l}\text { Massas d'água com presença } \\
\text { significativamente elevadas de nu- } \\
\text { trientes e matéria orgânica, com alto } \\
\text { comprometimento nos seus usos, } \\
\text { associado à presença exorbitante } \\
\text { de algas ou mortandades de peixes, } \\
\text { danificando até mesmo as ativi- } \\
\text { dades pecuárias nas proximidades. }\end{array}$ \\
\hline
\end{tabular}

Fonte: adaptado do site da Agência Nacional de Águas

(ANA, 2019).

Para realização dos cálculos de IET, utilizou-se as equações de 1 a 5 , dependendo do corpo hídrico ser lêndico ou lódico (CETESB, 2019)

Rios (lódico)

IET $(\mathrm{Cl})=10 \times(6-((-0,7-0,6 x(\ln \mathrm{Cl})) / \ln 2))-20$

IET $(\mathrm{PT})=10 \times(6-((0,42-0,36 x(\operatorname{In} \mathrm{PT})) / / \mathrm{ln} 2))-20$

Reservatórios (lêndico)

IET $(\mathrm{Cl})=10 \times(6-((0,92-0,34 \times(\operatorname{ln~Cl})) / \ln 2))$

IET $(\mathrm{PT})=10 \times(6-((1,77-0,42 \times(\ln \mathrm{PT})) / \ln 2))$

$$
\begin{aligned}
& \mathrm{Cl}=\text { clorofila } \mathrm{a}(\mu \mathrm{g} / \mathrm{L}) \\
& \mathrm{PT}=\text { fósforo }(\mathrm{MG} / \mathrm{L})
\end{aligned}
$$


Com os valores obtidos é realizado a seguinte formula final, para a conclusão do valor de IET:

$$
\text { IET }=[\operatorname{IET}(\mathrm{PT})+\mathrm{IET}(\mathrm{CI})] / 2
$$

\section{RESULTADOS E DISCUSSÃO}

Os resultados a seguir se referem ao período de maio de 2018 até fevereiro de 2019, demonstrados número em média das 4 coletas realizadas nesse período.

\subsection{Resultados de campo:}

No campo foram realizadas as análises através de triplicata, para que a média conclua um valor mais preciso para $\mathrm{pH}$, oxigênio dissolvido, condutividade elétrica e temperatura. Tabela 1.

Tabela1 - Resultados das análises em campo das quatro coletas.

\begin{tabular}{ccccc}
\hline Ponto & $\mathrm{Ph}$ & Temp. & $\mathrm{CE}$ & $\mathrm{OD}$ \\
\hline 1 & 6,66 & 21,5 & 89 & 13,2 \\
2 & 6,47 & 19,8 & 127,9 & 5,4 \\
3 & 7,25 & 23,1 & 153,3 & 12,2 \\
4 & 6,75 & 23,4 & 611,7 & 1,66 \\
\hline
\end{tabular}

No ponto quatro os altos valores da condutividade elétrica e o baixo valor do oxigênio dissolvido indicam que o corpo hídrico está altamente impactado pelo esgoto doméstico.

No laboratório foram processadas as seguintes análises de Turbidez (TU), Demanda Bioquímica de Oxigênio (DBO), E. coli, Fósforo Total (PT) e Nitrogênio Total (NT), tabela 2.

Tabela2 - Resultados das análises em laboratório após as quatro coletas.

\begin{tabular}{cccccc}
\hline Ponto & $\begin{array}{c}\text { TU } \\
\text { (UNT) }\end{array}$ & $\begin{array}{c}\text { DBO } \\
(\mathbf{m g ~ O 2 / L )})\end{array}$ & $\begin{array}{c}\text { E. coli } \\
(\mathbf{U F}- \\
\mathbf{C} / \mathbf{1 0 0 m} \mathbf{m})\end{array}$ & $\begin{array}{c}\text { PT } \\
(\mathbf{m g} / \mathbf{L})\end{array}$ & $\begin{array}{c}\text { Clorofila } \\
(\boldsymbol{\mu} \mathbf{g} / \mathbf{L})\end{array}$ \\
\hline 1 & 5,3 & 4,3 & $6,60 \mathrm{E}+03$ & 0,441 & - \\
2 & 10,8 & 10,9 & $6,60 \mathrm{E}+02$ & 0,45 & 4,4 \\
3 & 40 & 30,6 & $5,71 \mathrm{E}+03$ & 0,541 & 19,6 \\
4 & 180,9 & 289,3 & $9,50 \mathrm{E}+06$ & 2,669 & - \\
\hline
\end{tabular}

Os resultados das análises microscópicas e físico-químicas apontam que desde o primeiro ponto de coleta ao último há um crescimento do estado trófico das águas, devido ao acumulo de nutrientes em sua extensão, principalmente fósforo e Clorofila a (Tab. 2). Isso pode ser explicado, pois o corpo hídrico atravessa o zoológico, passando por vários viveiros, onde se acumula ração e dejetos dos animais.

Observou-se grande contaminação de E. coli, corroborado pelos valores de DBO, valores esses característicos de atividades antrópicas como esgoto e lixo doméstico, gerados e descartados aos arredores do córrego que se origina ali. Fia, et al. (2015) também chegou em resultados semelhantes em Lavras, MG.

\subsection{Resultados na análise Macroscópica:}

No quadro 4 são apresentadas as médias das avaliações, no entorno da nascente.

Quadro 4 - Resultados do impacto ambiental macroscópico para nascente.

\begin{tabular}{|l|l|}
\hline Parâmetro Macroscópico & Medição Obtida \\
\hline Cor da água & 3- Transparente \\
\hline Odor & 3- Inexistente \\
\hline Lixo ao redor & 2- Pouco \\
\hline Materiais flutuantes & 3- Inexistente \\
\hline Espumas & 3- Inexistente \\
\hline Óleos & 3- Inexistente \\
\hline Esgoto & 3- Inexistente \\
\hline Vegetação & 3- Muito presentes \\
\hline Uso por humano & 2- Esporádico \\
\hline Uso por animais & 1- Constante \\
\hline Acesso & 1- Fácil \\
\hline $\begin{array}{l}\text { Proximidade com área } \\
\text { urbana (metros) }\end{array}$ & 1- Menos de 50 \\
\hline
\end{tabular}

Quadro 5 - Classificação do grau de preservação da nascente, seguindo os resultados do quadro 1.

\begin{tabular}{|c|c|c|}
\hline $\begin{array}{c}\text { Resultado } \\
\text { calculado }\end{array}$ & $\begin{array}{c}\text { Pontuação Final } \\
\text { (pontos) }\end{array}$ & $\begin{array}{c}\text { Grau de } \\
\text { Preservação }\end{array}$ \\
\hline \multirow{2}{*}{28 pontos } & Entre 28 a 30 & Ruim \\
\cline { 2 - 3 } & Abaixo de 28 & Péssima \\
\hline
\end{tabular}

A partir da metodologia de GOMES; MELO; VALE, (2005), a nascente apresenta resultado de 28 pontos, sinalizando um grau ruim à péssima de preservação, devido ao fácil acesso. Funcionários do Zoológico realizam sazonalmente uma limpeza aos arredores da nascente, porém seria necessário um projeto de proteção nesse âmbito para a real preservação, já que seu efluente é uma fonte vital de alguns viveiros. 


\subsection{Resultado de IET}

Neste trabalho foram calculados os valores de fósforo para todos os pontos, diferente da clorofila a que não pode ser encontrada em corpos hídricos lóticos (rios) como no caso do ponto 1 e 4, porém foram lidos nos pontos 2 e 3 sendo estes lênticos (lagos/reservatórios), tendo assim que usar fórmulas diferenciadas.

Tabela 3 - Classificação de estado trófico para cada um dos pontos avaliados, ANA (2019).

\begin{tabular}{|c|c|c|c|c|}
\hline Pontos & $\begin{array}{l}\text { Valor de } \\
\text { IET } \\
\text { (CL) }\end{array}$ & $\begin{array}{l}\text { Valor de } \\
\text { IET } \\
\text { (PT) }\end{array}$ & $\begin{array}{l}\text { Valor de } \\
\text { IET } \\
\text { FINAL }\end{array}$ & $\begin{array}{l}\text { Categoria do } \\
\text { Nível trófico }\end{array}$ \\
\hline 1 & - & 65,6 & 65,6 & Supereutrófico \\
\hline 2 & 58,1 & 71,5 & 64,8 & Supereutrófico \\
\hline 3 & 65,4 & 72,6 & 69 & Hipereutrófico \\
\hline 4 & - & 74,9 & 74,9 & Hipereutrófico \\
\hline
\end{tabular}

O Ponto 1 e 2, mesmo sendo a nascente e seu primeiro reservatório, apresenta eutrofização, sendo classificado como Supereutrófico. Foi observado degradação significativa na nascente, alto valor de E.coli e fósforo. Roberti, Gomes e Bittencourt (2016) avaliaram quatorze nascentes de água, dentro dos limites urbanos do município de Muriaé-MG e todas encontram-se em um grau baixo de preservação, evidenciando poluição e contaminação.

$\mathrm{O}$ ponto 2 tem condições muito parecidas com o ponto 1 , mas há muito sedimento, e assim que é movimentado há aumento da turbidez da água.

O Ponto 3, o último e pequeno reservatório antes da saída do perímetro do zoológico, acumula todo o resíduo como ração, dejetos e outras formas de resíduos que os viveiros são capazes de produzir, por esse motivo foi classificado como Hipereutrófico. Esta característica inviabiliza todas as formas de uso dessa massa de água para qualquer fim.

Silva e Gouveia (2019) também observaram altos valores de nitrogênio, fósforo e clorofila-a, que são parâmetros indicadores de eutrofização, nas águas do Zoológico Quinzinho de Barros.

O Ponto 4 fica na área urbana, aproximadamente 100 metros da saída do zoológico, e apresenta altos valores de contaminação e pela análise do IET, pertence à classe Hipereutrófico. Observou-se odor bastante forte, acúmulo de lixo e a cor da água era acinzentada, características de local com contaminação de esgotos o que também foi constatado por Meneses et al (2016) e Dalmas et al (2016).

\section{CONCLUSÕES}

O córrego Ana Rita, que nasce no Zoológico Municipal de Guarulhos foi considerado eutrofizado, desde a nascente até o ponto urbanizado. Esse aporte de nutrientes pode ser explicado pela ração, dejetos do viveiro (dentro do Zoológico) e por lixo e esgoto lançado na área urbana.

Foi observado também que a nascente urbana, presente no Zoológico Municipal de Guarulhos - SP apresenta uma qualidade ambiental entre péssima e ruim devido à falta de proteção, fácil acesso de animais e proximidade com área urbana. 


\section{REFERÊNCIAS}

ANA - Agência Nacional de Águas. Portal da Qualidade das Águas Disponível em: http://portalpnqa. ana.gov.br. Acesso em: 18 de fev. 2019.

APHA, A. W. W. A.; W. E. F. Standard methods for the examination of water and wastewater, v. 22, 2012.

BRASIL. Lei no 12.651, de 25 de maio de 2012, Dispõe sobre a proteção da vegetação nativa; altera as Leis nos 6.938, de 31 de agosto de 1981, 9.393, de 19 de dezembro de 1996, e 11.428, de 22 de dezembro de 2006; revoga as Leis nos 4.771 , de 15 de setembro de 1965, e 7.754, de 14 de abril de 1989, e a Medida Provisória no 2.166-67, de 24 de agosto de 2001; e dá outras providências. Diário Oficial [da] República do Federativa do Brasil, Poder Executivo, Brasília, DF, 25 de maio de 2012.

BRASIL. Ministério do Meio Ambiente, Resolução CONAMA n ${ }^{\circ} 001$, de 23 de janeiro de 1986, estabelecerem as definições, as responsabilidades, os critérios básicos e as diretrizes gerais para uso e implementação da Avaliação de Impacto Ambiental como um dos instrumentos da Política Nacional do Meio Ambiente. Diário Oficial da União em 17 de fevereiro de 1986.

CETESB (São Paulo) - Apêndice D - Índices de qualidade das águas. Disponível em: https://cetesb. sp.gov.br/aguas-interiores/publicacoes-e-relatorios/ Acesso em: 11 de Mar. 2019.

DALMAS, F. B. et al. Reflexos do uso e ocupação do solo na qualidade da água do Rio Embu-Guaçu, Região Metropolitana de São Paulo. Geociências (São Paulo), v. 34, n. 1, p. 130-144, 2016. Disponível em: http://www.ppegeo.igc.usp.br/index.php/GEOSP/article/view/8496. Acesso em: 30 set. 2019.

ESTEVES, F. A. Fundamentos de limnologia 3. ed. São Paulo: Interciência, 2011.

FIA, R. et al. Qualidade da água de um ecossistema lótico urbano. Revista Brasileira de Recursos Hídricos, v. 20, n. 2, p. 267-275, 2015. Disponível em: https://www.researchgate.net/profile/Fatima_Fia/ publication/305306771_Qualidade_da_agua_de_ um_ecossistema_lotico_urbano/links/57cdb3e008ae83b37460e30f/Qualidade-da-agua-de-um-ecossistema-lotico-urbano.pdf. Acesso em: 11 out. 2019.
GOMES, P. M.; MELO, C. VALE, V. S. Avaliação dos impactos ambientas em nascente na cidade de Uberlândia - MG: análise macroscópica. Revista sociedade e natureza. Uberlândia, 17 (32), p. 103 - 120, jun. 2005.

MENEZES, J. P. C. et al. Relação entre padrões de uso e ocupação do solo e qualidade da água em uma bacia hidrográfica urbana. Revista de Engenharia Sanitária e Ambiental, v. 21, n. 3, p. 519-534, 2016. Disponível em: file:///C:/Users/280700001/Downloads/Qualidade_da_agua_de_um_ecossistema_lotico_urbano.pdf Acesso em 30 de set 2019.

ROBERTI, H. M.; GOMES, E. R.; BITTENCOURT, A. H. C. Estado de conservação das nascentes no perímetro urbano da cidade de Muriaé-MG. Revista Científica da Faminas, v. 4, n. 1, 2016.

ROCCO, E. Implicações jurídicas da implantação da esteção ecologica do Tanque- Grande. 2015. 100 f. Dissertação (Mestrado em Análise Geoambiental) - Universidade UNG, Guarulhos. 2015.

SÃO PAULO, decreto $n^{\circ} 10.755$, de 22 de novembro de 1977, Dispõe sobre o enquadramento dos corpos de água receptores na classificação prevista no Decreto $n^{\circ} 8.468$, de 8 de setembro de 1976 e dá providências correlatas. Governo do estado de São Paulo, Poder Executivo, São Paulo, SP, 23 de novembro de 1977.

SILVA, C. O. F.; GOVEIA, D. Avaliação da qualidade ambiental de corpos hídricos urbanos utilizando análise multivariada. Interações, Campo Grande, v. 20 , n. 3, p. 947-958, 2019. Disponível em: http:// dx.doi.org/10.20435/inter.v0i0.1832. Acesso em: 07 out. 2019.

TUCCI, C. E. M. Urbanização e Recursos Hídricos. IN: BICUDO, C. E. M.; TUNDISI, J. G.; SCHEUENSTHUL, M. C. B. Águas do Brasil: análises estratégicas. São Paulo: Instituto de Botânica, 2010. p.113-132.

WETZEL, R. G.; LIKENS, G. E. Limnological analyses. New York: Springer-Verlag, 1991. 